\title{
Surfaces
}

\section{MISSION IMPOSSIBLE : LES INTELLECTUELS SANS LA CULTURE}

\section{Bruce Robbins}

Volume 2, 1992

ACTES DU COLLOQUE « REPENSER LA CULTURE » ACTS OF THE CONFERENCE "RETHINKING CULTURE"

URI : https://id.erudit.org/iderudit/1065232ar

DOI : https://doi.org/10.7202/1065232ar

Aller au sommaire du numéro

Éditeur(s)

Les Presses de l’Université de Montréal

ISSN

1188-2492 (imprimé)

1200-5320 (numérique)

Découvrir la revue

Citer ce document

Robbins, B. (1992). MISSION IMPOSSIBLE : LES INTELLECTUELS SANS LA

CULTURE. Surfaces, 2. https://doi.org/10.7202/1065232ar
Résumé de l'article

Élaboration d'une notion critique de la vocation de l'intellectuel, qui met en cause l'idéologie du désintérêt et affirme que l'intellectuel radical doit prendre conscience des rapports de pouvoir implicites dans sa prétention de parler au nom du peuple.
Copyright (c) Bruce Robbins, 1992

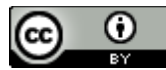

Ce document est protégé par la loi sur le droit d'auteur. L'utilisation des services d’Érudit (y compris la reproduction) est assujettie à sa politique d'utilisation que vous pouvez consulter en ligne.

https://apropos.erudit.org/fr/usagers/politique-dutilisation/ 


\author{
ACTES DU COLLOQUE \\ "REPENSER LA CULTURE" \\ MISSION IMPOSSIBLE: \\ LES INTELLECTUELS SANS LA CULTURE
}

Bruce Robbins

Cette conférence a été donnée au colloque Repenser la culture

qui s'est tenu à l'Université de Montréal, les 3, 4 et 5 avril 1992.

Les organisateurs tiennent à remercier, pour leur soutien financier:

le Conseil de Recherche en Sciences Humaines du Canada

la Faculté des Arts et des Sciences, Université de Montréal

le Vice-rectorat à l'enseignement et à la recherche, Université de Montréal

le Département de Littérature comparée, Université de Montréal

Alitalia

RÉSUMÉ

Élaboration d'une notion critique de la vocation de l'intellectuel, qui met en cause l'idéologie du désintérêt et affirme que l'intellectuel radical doit 
prendre conscience des rapports de pouvoir implicites dans sa prétention de parler au nom du peuple.

\begin{abstract}
A critique of the vocation of the intellectual, which argues against the pretension to disinterest and insists that the radical intellectual must take account of the power-relations implicit in the claim to speak for the people.
\end{abstract}

J'ai donné à ces remarques le titre de "Mission impossible," et ceci pour deux raisons: premièrement, parce que c'est plus ou moins en ces termes que je me suis fixé la tâche de vous parler en français; deuxièmement, parce que mon sujet d'aujourd'hui est la mission des intellectuels, ou plus précisément la façon dont ils s'autorisent, se donnent une mission alors que le vocable même de "mission" paraît démodé, inutilisable, bref, impossible.

La question que j'aimerais poser -- sans trop savoir comment y répondre - -est la suivante: si la culture a été le capital des intellectuels, selon la métaphore bien connue de Pierre Bourdieu, si les intellectuels se sont servis de la culture pour se définir, se légitimer, se reproduire, bref, si la culture a été la base ou le fondement des intellectuels, que se passe-t-il dès le moment -- cela semble être notre moment -- où les intellectuels acceptent cette analyse et son bien-fondé, et où le concept de culture devient la cible d'une critique plus ou moins généralisée? Comment expliquer le succès de cette critique auprès des intellectuels auxquels elle semble enlever les fonds et le fondement? Comment doivent-ils se concevoir, ou sont-ils censés se placer, quand ils sont ceux qui reconnaissent et qui dénoncent le capital culturel? Devons-nous accepter l'image paradoxale d'un dessaisissement volontaire par des intellectuels de leur capital? Ou les intellectuels sont-ils appuyés par une économie pas encore reconnue comme telle, une forme de capital postculturel qui les fait vivre clandestinement? Sur quelle base se reposent-ils à partir du moment où ils refusent ou délaissent leur base culturelle?

Il est possible, bien sûr, que les intellectuels ne soient tout simplement pas conséquents quand ils dénoncent le capital culturel tout en en bénéficiant. Se rendre compte de cette possibilité, c'est une précaution contre ce que Chantal Mouffe et Ernesto Laclau appellent le "logicisme": une certaine volonté de flotter, en théorisant, au-dessus des contradictions sociales et 
historiques (on pourrait dire que la même accusation touche leur propre projet). J'offre la problématique de la mission ou de la vocation comme moyen d'éviter ce piège, comme un croisement relativement délimité entre les idées et les pratiques sociales: un lieu où le vacillement de l'autorité de la culture prend la forme d'un dérèglement ou d'un /pp. 5-6/ changement de cap dans la reproduction ou le recrutement des intellectuels.

Selon l'analyse classique de Raymond Williams, le concept de culture s'est défini, pendant et depuis la période romantique, comme réaction contre la révolution industrielle et comme contrepartie de la division exacerbée du travail. Il désigne un tout, une intégrité idéale, à la fois individuelle et collective, qui s'oppose à l'instrumentalisation et à la parcellisation de la société capitaliste, tout en la soignant. Petit à petit, à travers les visions nostalgiques de Matthew Arnold, de T.S. Eliot et de leurs successeurs, la culture devient ainsi une façon de persuader les divers détenteurs de fonds de l'État -- les donateurs privés et les fondations -- qu'une société Waste Land ("terrain vague") commercialisée, vidée par la technologie de toute valeur authentique, est malade, malade d'un manque de valeurs et de traditions que les intellectuels seuls ont maintenues et que les intellectuels seuls peuvent maintenant fournir. Ainsi s'explique en grande partie la montée, au sein d'une société de plus en plus thérapeutique, de la discipline de la critique littéraire, avec le paradoxe que c'est justement cette aspiration vers la transcendance de la division du travail qui a permis aux critiques littéraires de s'inscrire au sein de la division du travail, en se constituant en corps de métier reconnu parmi les autres métiers. Et ainsi s'explique aussi la vocation, l'interpellation de l'intellectuel, en tant qu'individu, qui peut donc se considérer non seulement comme une personne qui reçoit et transmet l'héritage culturel, mais comme un thérapeute et même un sauveteur, comme l'émetteur-récepteur de la culture dans un état perpétuel d'urgence (où la communication entre présent et passé se fait difficilement). Sa vocation, c'est l'appel d'une société qui a besoin de lui.

Il est évident que cette logique de légitimation est maximisée dans la mesure où la culture reste une culture non vécue. Ce n'est pas parce qu'on lit Shakespeare qu'on a besoin de commentaires sur lui, mais parce qu'on ne le lit pas; en tant que dépôt de valeurs, la tradition culturelle prend ellemême une valeur inversement proportionnelle à sa persistance comme force sociale vivante. Mais si les intellectuels ont besoin de vides à remplir, de problèmes sociaux à résoudre, et si la culture-comme-savoir a été l'une de ces solutions par lesquelles les intellectuels se sont installés dans la société, comment expliquer chez les intellectuels le passage si récent vers la culture non vécue vers la culture vécue, ordinaire, de masse, ethnographique?

Une réponse possible, c'est qu'après tout, en dépit de la montée des cultural studies à l'université et de la fureur de la presse, très peu a changé. Le mouvement d'une conception "haute", "classique", "universelle" de la 
Culture, vers la culture "ordinaire", "plurielle", "ethnographique", peut très bien se faire de sorte que certains éléments-clés de la culture restent en place, et avec eux la conception héroïque de l'intellectuel qu'ils appuyaient. L'historien de l'anthropologie, James Clifford, parle du paradigme de sauvetage (salvage paradigm ). Dans ce cadre de la vocation, l'ethnologue saisit la culture authentique des autres, la culture non occidentale, à l'instant même où, entrée en contact avec nous, avec l'Occident, cette culture est près de perdre son authenticité et son identité, c'est-à-dire de disparaître en tant que telle. L'ethnologue qui se prête à un tel scénario, arrachant une culture au néant qui la menace, se joint, ce faisant, à la même équipe de secours culturel rassemblée par T.S. Eliot au service des critiques littéraires, à la seule différence que dans ce cas l'acte critique de sauvetage prend place dans l'espace plutôt que dans le temps. L'intellectuel reste le sauveteur héroïque des espèces culturelles en voie de disparition, et l'obligation demeure de penser que la culture est toujours en voie ou en danger de disparition, car c'est ainsi que l'intellectuel sauvegarde la mesure certaine de son héroïsme.

Mais là encore, il faut constater que la critique de Clifford ne représente pas une voix unique et solitaire; au coeur même de l'essor des cultural studies, elle devient de plus en plus le sens commun des intellectuels qui se prononcent sur la culture. Le paradigme de sauvetage ne peut pas expliquer le fait que la critique du sauvetage se répande un peu partout. Il ne peut pas expliquer non plus le sens de la vocation qui ressort de la culture de masse, la culture "basse" ou populaire qui est visiblement et incontestablement vivante. En ce qui concerne le respect croissant pour la culture populaire chez les intellectuels, qui jusqu'à présent n'en parlaient pas très respectueusement, il faut regarder ailleurs.

Andrew Ross, dans son livre récent No Respect: Intellectuals and Popular Culture, fait l'analyse du mépris que la plupart des intellectuels du type classique ont démontré à l'égard de la culture populaire. L'intérêt derrière ce mépris n'est pas difficile à comprendre: si on admet que la culture populaire mérite bien le nom de culture au même titre que la soi-disant culture "universelle", elle échappe à leur domaine d'expertise et menace de faire éclater leur monopole. Selon Ross, "in an age of expert rule, the popular is perhaps one field in which intellectuals are least likely to be experts"[1]. Nous revenons donc à notre question: quel genre d'expertise et quelle justification revendiquent-ils maintenant? Sur quoi les intellectuels se basent-ils quand ils se défont de leur base habituelle?

On pourrait penser que chez des critiques de gauche comme Ross, Edward Said, Gayatri Spivak, Paul Bove, et. al., la vocation nouvelle prendrait la forme d'une démocratie élargie de la représentation. Mais comme nous le savons tous déjà, l'élargissement démocratique du champ d'objets que la critique examine n'a pas été accompagné ni exprimé par un regain d'intérêt pour le lexique de circonscriptions électorales. Au contraire, au lieu de puiser l'autorité dans la représentation de ceux qui avaient été privés de 
représentation, la mode a été à l'interrogation plus ou moins hostile des intellectuels par les intellectuels. L'interrogation surtout des procédés par lesquels les intellectuels ont servi leurs propres intérêts en s'appuyant sur des fictions du "peuple" (en d'autres termes, de la légitimation). La critique de la culture s'est poursuivie, même chez des critiques qu'on considère comme représentants des cultures du Tiers Monde jusqu'ici oubliées ou marginalisées, en même temps qu'une critique de tout effort de représentation ou de légitimation par lequel les intellectuels se donneraient une position sociale en tant que représentants de la culture, même de la culture la plus populaire.

Je cite Edward Said: "la culture a été utilisée essentiellement non pas comme un terme coopératif ou communautaire mais comme un terme d'exclusion"; il est donc temps, dit-il, d'accepter "le grand fait moderne ou postmoderne, de se mettre à l'extérieur des cultures" (the standing outside of cultures ).[2] Gayatri Spivak a parlé avec son éloquence habituelle contre la procédure selon laquelle l'intellectuel se pose comme défenseur des intérêts des non-représentés, transmetteurs des voix jusque-là réduites au silence. À propos du rôle des intellectuels envers les cultures du Tiers Monde, elle parle du "risque d'effacer 'l'indigène' et de s'avancer comme 'le vrai Caliban'", "en prétendant qu'on est Caliban."[3] Ceux qui revendiquent la marginalité après dix ans d'études post-coloniales, écrit-elle, se trouvent peut-être embrouillés dans "the old scenario of empowering a privileged group or a group susceptible to upward mobility as the authentic inhabitants"[4]. La critique la plus directe de cette logique de légitimation se trouve peut-être dans l'avant-dernier livre de Paul Bové, Intellectuals in Power. En établissant une conception de la culture en parallèle avec l'État, écrit-il, les intellectuels assurent leur propre position, légitimation, pouvoir: "The essential requirement of 'culture' is that it parallel, reinforce, and embody the political regulations of civil society and the state."[5] Ce projet vit et prospère toujours, selon Bové, même chez des intellectuels d'opposition comme Edward Said, parce qu'il facilite "la légitimation de toutes les entreprises critiques"; "to justify itself, oppositional intellectual practice can and almost must reinscribe the figure of the leading intellectual as representative" (279). En tant que représentant de n'importe quoi, sauf de lui-même, l'intellectuel ne mérite que le soupçon.

C'est peut-être le soupçon de notre ère. Mais ce constat ne résoud en aucune manière la question de départ: où l'intellectuel soupçonneux si caractéristique de cette ère se tient-il? Quand il soupçonne l'intérêt derrière ces démarches intellectuelles, prétend-il agir d'une façon désintéressée? Sinon, quel intérêt peut-il avouer? Par quelle logique d'intérêt, d'autorité, de vocation est-il interpellé?

Comme réponse très partielle, j'aimerais ébaucher ici une liste très approximative parce que je ne suis pas capable d'en faire plus, mais aussi parce qu'il me semble déjà utile de pouvoir en faire autant: à savoir 
proposer qu'il existe des logiques vocationnelles possibles au-delà de la représentation de la culture.

Premièrement: un rôle que l'intellectuel pourrait se donner, c'est de démontrer, contrairement à ce qu'on pourrait penser, qu'en ce qui concerne la culture populaire on n'est pas en présence du peuple. Dans un essai intitulé "Can the Subaltern Speak?", Gayatri Spivak doute non seulement du droit des intellectuels à se poser en repré sentants légitimes, mais aussi de la capacité des opprimés, au milieu de leur oppression, d'articuler une volonté affranchie, unifiée, et cohérente que l'intellectuel puisse ensuite représenter. Répondant à cet argument, Benita Parry accuse Spivak de s'élever ou de s'avancer personnellement au prix des subalternes. Chez Spivak, écrit-elle, "the disparaging of nationalist discourses of /pp. 10-11/ resistance is matched by the exorbitation of the role allotted to the postcolonial woman intellectual." [6]

Examinons cette logique. Parry n'a pas tort, je crois, de prétendre que nier la capacité des opprimés de s'exprimer pleinement et librement peut s'accompagner de l'affirmation des droits et des privilèges de l'intellectuel qui est supposé s'exprimer mieux. Mais l'affirmation de l'intellectuel peut très bien se faire aussi en célébrant la capacité des opprimés de s'exprimer -- une affirmation faite sinon à leurs frais, du moins sur leur dos. Donc, le révélation d'un intérêt personnel quelconque ne doit pas être comprise, ni présentée, comme une critique destructrice. L'intellectuel ne peut pas échapper à l'intérêt personnel, et se lancer des fleurs de ce genre ne sert donc pas à grand chose.

La question bien plus intéressante ici est de savoir comment expliquer que le refus de la représentation puisse produire des bénéfices pour l'intellectuel qui semble ainsi se priver inutilement de base. Je vois deux possibilités. D'abord, on pourrait peut-être considérer ce refus comme une version modifiée de la culture elle-même, ainsi que de sa logique propre de légitimation. Ce refus passe évidemment par la critique ou l'auto-critique de l'intellectuel, qui s'insurge contre lui-même ou contre ses confrères pour abus de privilèges. Dans une société capitaliste, intéressée, instrumentalisée, celui qui s'accuse d'égoïsme montre par là même qu'il n'agit pas d'une façon complètement intéressée ou instrumentale; il se met en dehors des lois du marché. C'est-à-dire qu'il fait ce qu'étaient censés faire les littéraires, la littérature, la culture; du moins, il offre les mêmes preuves de vertu. En ce sens, la théorie littéraire, devenue (comme certains s'en plaignent) une nouvelle routine, s'installerait malgré tout dans la même lignée que la routine familière de la critique littéraire, avec ses rites pieux de pureté, de marginalité, de désintéressement. 
La deuxième possibilité, liée à la première, serait que l'auto-critique ne fonctionne pas comme refus de la représentation, mais à un autre niveau comme revendication de la représentation, revendication indirecte mais (pour cette raison même) fortifiée. La logique serait plus ou moins celle-ci: en niant mon droit de représenter le peuple, je prouve que je le respecte plus que vous, donc que je le représente mieux que vous. Dans cette optique, ce qu'on a l'habitude de voir comme une critique de la représentation, un effort pour la démanteler ou s'en défaire, serait plus exactement conçue comme une recherche de la représentation. Ou plutôt, cet effort aurait un tout autre visage, celui exposé récemment pendant les guerres mediatiques à propos du Political Correctness . La Droite critique l'Université parce qu'elle est trop théorique, trop fermée aux gens ordinaires, et, simultanément (sous l'influence des mêmes enseignants, les tenured radicals des années soixante), parce qu'elle laisse entrer trop de femmes, trop de Noirs, trop de gens sans préparation adéquate, etc.: donc, pour excès de démocratie parce qu'elle est trop ouverte aux gens ordinaires. La théorie n'a pas toujours su quoi faire de ses liens avec les groupes et les mouvements agissant au nom de l'identité sexuelle, raciale, etc. La Droite pourrait donc nous apprendre quelque chose.

Mais avant de réhabiliter la représentation, essayons une autre hypothèse. Selon Spivak, dans "Can the Subaltern Speak?", ce qui empêche les subordonnés de trouver leur voix, c'est la puissance du Discours. Cette puissance formatrice et déformatrice, qui depuis Foucault a eu tant de succès auprès des intellectuels nouveau-type, pourrait-elle jouer quelque rôle oblique et insidieux dans leur légitimation? En d'autres termes, peut-on recevoir une vocation d'un faux dieu aussi bien que d'un vrai? Le Discours, cette force énorme et presque inimaginable qui interdit ou déforme la parole du subalterne, semble bien régner partout ici-bas. L'intellectuel dont le savoir professionnel traite et dépend du Discours, qui se soumet au Discours et qui le connaît de façon intime, cet intellectuel devient en quelque sorte le porte-parole terrestre d'un dieu hérétique et prophète à la fois. On pourrait peut-être en dire autant du terme d'Idéologie chez Althusser. C'est en effet une sorte de parodie cruelle de la vocation religieuse qu'Althusser nous offre en proposant son image célèbre de l'"interpellation", la vocation par l'Idéologie, ce cri de la police dans lequel l'individu se reconnaît sans savoir pourquoi, et vers lequel il se tourne fidèlement. L'Idéologie d'Althusser, comme le Discours de Foucault, porte une majuscule implicite; celle-là même, peut-être, que la Culture a si récemment perdue. Tous les deux sont (ou du moins sont reçus comme) des dieux omniprésents, omniscients, et tout-puissants. S'ils sont aussi malveillants, il est possible que la vocation exige la toute-puissance et l'accepte même accompagnée de la malveillance. Car comme le sublime, elle aussi semble passer au-delà du bien et du mal; ce qui compte, c'est le pouvoir, analogue au sublime, le pouvoir mobilisateur de lier les efforts de l'intellectuel à l'horizon absolu du concevable et du faisable. 
Ce principe, la vocation retrouvée à travers des divinités conceptuelles à la fois toute-puissantes et malveillantes, on pourrait le retracer un peu partout dans le nouveau style intellectuel. On pourrait parler, par exemple, de l'impérialisme, compris par les études culturelles à l'instar de la culture, c'est-à-dire comme un tout garantissant d'une façon théologique que le refoulé reviendra toujours se montrer dans les textes de la culture impériale. Savons-nous vraiment d'avance que les signes de l'Empire ne peuvent pas faire autrement que de se manifester? Sommes-nous convaincus qu'il est impossible que ces signes soient vraiment absents des textes anglais des années 1840, par exemple? Même si ces textes ne parlent pas ouvertement de la famine irlandaise si proche, est-il possible que leurs auteurs n'aient pas enregistré cet événement impérialiste de quelque manière que ce soit? Ou n'est-ce pas faire du système impérial une sorte de dieu, afin de se donner, en tant qu'interprètes, du travail honorable et sans fin?

Mais j'épargnerai les exemples pour passer à quelques reflexions, en guise de conclusion, sur la métaphore du "capital culturel" chez Bourdieu, sur l'État, et sur la sexualité.

Me trouvant ici dans une situation "linguistique", je ne peux pas me refuser le plaisir de citer Pierre Bourdieu en anglais. Dans une conversation (en anglais) avec Terry Eagleton publiée dans le dernier numéro de la New Left Review , Bourdieu défend le concept de "capital culturel" contre la mode des cultural studies : "It is a form of dominant chic among intellectuals to say 'Look at these cartoons,' or some other cultural item, 'do they not display great cultural creativity?' Such a person is saying, 'You don't see that, but I do, and I am the first to see it.' The perception may be valid; but there is an overestimation of the capacity of these new things to change the structure of the distribution of symbolic capital. To exagerate the extent of change is, in a sense, a form of populism. You mystify the people when you say 'Look, rap is great'... you forget what remains the dominant form, and that you still can't realize symbolic profits from rap, in the main social games." [7]

Ce qui me frappe le plus ici, c'est la volonté de croire, contre toute évidence, qu'il a existé et qu'il existe toujours une seule et unique "forme dominante" de la culture, seule source de bénéfices qui compte dans les "jeux sociaux". Une volonté non seulement de défendre les bénéfices que Bourdieu a tirés lui-même de la découverte (ou l'invention) de cette forme dominante, c'est-àdire de la métaphore de capital culturel, mais aussi de réduire l'autre -- celui qui pratiquerait des cultural studies -- à l'intérêt personnel ("Vous ne le voyez pas, mais moi je le vois, et je suis le premier à le voir"), intérêt personnel qui apparemment n'a pas de place chez lui. La stratégie est claire: accroître sa divinité malveillante, c'est amoindrir et cacher son carrièrisme. Le monothéisme de Bourdieu va pieusement de pair avec une prétention, tout aussi théologique, au désintéressement. 
Notons en passant que ce culte négatif du capital culturel, qui dépasse largement le champ de ceux qui prennent le travail de Bourdieu très au sérieux, trahit sa propre métaphore. Pour Bourdieu, la culture est vide. Le contenu du capital culturel, fixé par l'État, est complètement arbitraire.[8] Il ne correspond qu'au but de maintenir les privilèges de la classe dominante. Mais si on n'avait pas appris le contraire ailleurs et autrement, par exemple, lors de tous les débats dans la presse à propos des études culturelles, on pourrait l'apprendre chez Marx. L'origine du capital, selon Marx, c'est la plus-value, c'est-à-dire le travail et les rapports entre la capital organique et inorganique, le capital qui ressort directement du travail et le capital qui a pris la forme des machines, des bâtiments, des institutions. Or, ces rapports ne sont absolument pas arbitraires. Revenant donc à notre métaphore, il faudrait dire que le capital culturel est aussi composé d'éléments qui viennent d'en bas, pour ainsi dire, et que le capital culturel est, lui aussi, radicalement hétérogène. Modeste champ de bataille, le capital culturel ne peut donc pas servir de divinité à des fins vocationnelles, et il ne peut pas non plus garantir le désintéressement de ses interprètes. Finalement, le populaire d'un coté, et l'égoïsme intellectuel de l'autre, que Bourdieu stigmatise également, montent ensemble sur la scène de la vocation.

Je précise: mon intention ici n'a pas été de légitimer la légitimation. J'ai voulu y réintroduire un peu de jeu, relâcher un peu sa fermeture tautologique. La légitimation des intellectuels, telle qu'on en parle d'habitude, suggère un cercle fermé où l'État se reproduit en faisant appel à des valeurs sociales trop bien calquéessur les valeurs de l'État même. Mais tout comme l'expression de "capital culturel", le mot "reproduction" témoigne d'un certain excès. La reproduction exige le désir; le désir, l'érotisme. En passant de la reproduction à la vocation, on arrive à la séduction, avec ce que cela comporte de risque, d'aventure, de distance par rapport aux objets familiers et rassurants. Le mot "séduction" est évidemment dangereux. Mais puisqu'il n'est pas possible de se passer de légitimation, mieux vaut essayer de la repenser sous cette forme risquée et érotisée. Puisqu'on ne peut pas éliminer tout à fait la loi de la légitimation, on peut peut-être essayer de la pratiquer d'une façon déloyale, illégitime. Et parce qu'il faut que les intellectuels abandonnent enfin la fiction de leur désintéressement, il faudra commencer à repenser leur propre intérêt sans cynisme; surtout, sans ce que le cynisme peut comporter de confortable et d'intéressé.

\section{Bruce Robbins}

Department of English

\section{Rutgers University}


Surface Page d'Acceuil/Home Page

[1]Andrew Ross, No Respect: Intellectuals and Popular Culture (NY: Routledge, 1989), pp. 231-32.

[2]Edward Said, "Media, Margins, and Modernity" in Raymond Willimas, The Politics of Modernism: Against the New Conformists, ed. Tony Pinckney (London: Verso, 1989), p. 196.

[3]Gayatri Chakravorty Spivak, "Three Women's Texts and a Critique of Imperialism," Critical Inquiry, 12 (Autumn 1985), pp. 243-61: "the risk of effacing 'the native' and stepping forth as 'the real Caliban,'" "claiming to be Caliban" (245).

[4]Gayatri Chakravorty Spivak, "Poststructuralism, Marginality,

Postcoloniality, and Value," Literary Theory Today, eds. Peter Collier and Helga Geyer-Ryan (Ithaca: Cornell UP, 1990), p. 223: "As a result of a decade of colonial discourse studies percolating into disciplinary pedagogy and its powerful adjuncts, and of the imbrication of techniques of knowledge with stratetgies of power, who claims marginality in the larger post-colonial field? What might this have to do with the old scenario of empowering a privileged group or a group susceptible to upward mobility as the authentic inhabitants?"

[5]Paul Bové, Intellectuals in Power (NY: Columbia UP, 1986), pp. 252-53.

[6]Benita Parry, "Problems in Current Theorires of Colonial Discourse,", Oxford Literary Review, 8 (1986), p. 35.

[7]Pierre Bourdieu and Terry Eagleton, "In Conversation: Doxa and Common Life," New Left Review, 191 (January/February 1992), p. 119.

[8]Pierre Bourdieu et Jean-Claude Passeron, La Reproduction (Paris: Minuit, 1970). Jacques Ranciere commente: "Toute l'opération se joue sur un seul concept: l'arbitraire," "L'éthique du sociologue", Collectif "Révoltes

Logiques" L'Empire du sociologue (Paris: La Découverte, 1984), p.27. 